Investigaciones Fenomenológicas, vol. Monográfico 7, 2018, 151-168 e-ISSN: $1885-1088$

\title{
La nOción de ciencia de la cultura en Alfred Schutz SEGÚN LA VISIÓN DE LESTER EMBREE
}

\author{
ALFRED SCHUTZ'S NOTION OF CULTURAL SCIENCE \\ IN LESTER EMBREE'S VIEW
}

\author{
Roberto J. Walton \\ Universidad de Buenos Aires \\ grwalton@fibertel.com.ar
}

Resumen: El artículo expone la interpretación y ampliación realizada por Embree respecto de las ideas de Schutz sobre las ciencias de la cultura. Se ocupa en primer lugar de las características generales de todas las ciencias de la cultura en su tarea de analizar el mundo de la vida como un mundo intersubjetivo e histórico estructurado de acuerdo con coasociados, contemporáneos, predecesores y sucesores. Luego examina los tres partes constituyentes principales que Embree destaca en cada una de ellas: la definición de la disciplina, sus conceptos básicos y sus reglas de procedimiento. Por último, se consideran los puntos de vista de Embree sobre la relación entre metodología y filosofía. Su tesis es que científicos y filósofos pueden encontrarse en una interacción mutua en el ámbito de la teoría de la ciencia que puede ser a la vez filosófica y científica.

Palabras clave: Ciencias de la cultura. Mundo de la vida. Conceptos básicos. Metodología.

\begin{abstract}
The article is a presentation of Embree's interpretation and development of Alfred ideas on the cultural sciences. It deals first with the general characteristics of all cultural sciences in their task of analyzing the lifeworld as an intersubjective and historical world structured according to consociates, contemporaries, predecessors, and successors. Then it examines the three main constituent parts that Embree highlights in each of them: the definition of the discipline, its basic concepts, and its rules of procedure. Finally, Embree's views on the relationship between methodology and philosophy are considered. His thesis is that scientists and philosophers can meet in a mutual interplay in the realm of a theory of science that can be both philosophic and scientific.
\end{abstract}

Keywords: Cultural sciences. Lifeworld. Basic concepts. Methodology.

Lester Embree promovió la idea de que el quinto estadio de la fenomenología -luego de las etapas realista, trascendental, existencial y hermenéuticatendría como uno de sus problemas centrales el desarrollo de una teoría de la 
cultura1. Y afirmó: "Mi conjetura es que el tema general sería entonces la vida humana tanto colectiva como individual en el mundo socio-histórico, un tema que es idóneo para una filosofía de la cultura reflexivo-descriptiva" ${ }^{2}$. En una de sus últimas obras se refiere extensamente a esta cuestión sobre la base de ideas expuestas por Alfred Schutz ${ }^{3}$. La obra no solo es una presentación sistemática de las ideas de Schutz sino también una interpretación de ellas en el sentido de una visión adicional propia que elabora posibles modos de comprensión y de desarrollo ulterior mediante el señalamiento de problemas y la apertura de caminos de solución. Por esta razón, en el título, se ha preferido el término "Schutziano" a la expresión "de Schutz"4. Embree procura esclarecer el siguiente problema: "Definiciones disciplinares, conceptos básicos y procedimientos distintivos son claramente los aspectos de la ciencia que interesaron profundamente a Schutz, pero, ¿de qué manera y con qué fin"5. Toda la obra es un esfuerzo por explicitar las modalidades y las metas de las ciencias culturales con consideraciones, en la primera parte, sobre la economía, la jurisprudencia, la ciencia política y la sociología tal como se presentan en la obra de Schutz, y con la complementación personal, en la segunda parte, en que "el enfoque de Schutz es llevado en su espíritu, pero más allá de su letra, a otras disciplinas"6 como la arqueología, la antropología cultural, la enfermería y la psicoterapia. Por último, en la tercera parte, la obra se ocupa de estudios filosóficos complementarios. En suma: Embree aporta una visión personal sobre las ciencias culturales o ciencias humanas respecto de las cuales se destacan los siguientes temas fundamentales: (i) los rasgos distintivos de todas las ciencias culturales; (ii) la

\footnotetext{
${ }^{1}$ Cf. Lester Embree y J. N. Mohanty, "Preface", Encyclopedia of Phenomenology, Contributions to Phenomenology 18, Dordrecht/Boston/London: Kluwer Academic Publishers, 1997, pp. 5s. Mi relación con Lester Embree se inició como participante en esta obra, y mi último encuentro personal con él se produjo en ocasión del Congreso "Life-World, Politics and Power", organizado por el International Alfred Schutz Circle for Phenomenology and Interpretative Social Science, y realizado en Buenos Aires en abril de 2014.

${ }^{2}$ Lester Embree, "La continuidad de la fenomenología: ¿un quinto período?", Escritos de Fenomenología 37-38 (2000), p. 28. Este texto fue leído el 24 de septiembre de 1999 en el X Encuentro Nacional de Fenomenología, organizado por la Sección Fenomenología y Hermenéutica del Centro de Estudios Filosóficos Eugenio Pucciarelli de la Academia Nacional de Ciencias de Buenos Aires. Su generosa y espontánea participación en este Encuentro fue uno de los innumerables ejemplos que reflejaron su admirable e incansable tarea de promover la fenomenología mediante la ampliación de sus horizontes de problemas, el aliento a sus cultores en todas las regiones del mundo y el establecimiento de sólidas y fructíferas relaciones entre ellos.

3 Lester Embree, The Schutzian Theory of Cultural Sciences, Cham/Heidelberg/New York/Dordrecht/London: Springer, 2017.

${ }^{4}$ Cf. ibid, p. viii.

${ }^{5}$ Ibid., p. 3.

${ }^{6}$ Ibid., p. 9; cf. p. 173.
} 
definición de cada una de estas ciencias como disciplina; (iii) sus conceptos básicos; (iv) su metodología; y (v) la relación con la filosofía.

\section{RASGOS DISTINTIVOS DE LAS CIENCIAS DE LA CULTURA}

El análisis de las ciencias presenta en primer lugar una diferencia genérica entre las ciencias de la naturaleza y, según las denominaciones utilizadas por Schutz, las ciencias de la cultura, ciencias del espíritu o ciencias sociales. Todas las ciencias culturales se ocupan de aspectos del mundo de la vida históricosocial. Se trata del mundo con el que nos enfrentamos primariamente y respecto del cual la naturaleza ocupa una posición derivada. Según Schutz, el concepto de naturaleza es "una abstracción que, en principio y por supuesto legítimamente, excluye personas y su vida personal y todos los objetos de la cultura que se originan como tales en la actividad humana práctica"7. Aquello de lo que hacen abstracción las ciencias de la naturaleza es precisamente tema de las ciencias sociales o culturales. Embree destaca que, para Schutz, las ciencias culturales se refieren a objetos culturales en un sentido amplio y caracterizados por tener un sentido: "Esta textura de sentido $-y$ esto distingue el reino de la cultura del reino de la naturaleza - se origina en y ha sido instituido por acciones humanas, las nuestras y las de nuestros congéneres, contemporáneos y predecesores" ${ }^{\prime}$.

Si bien es una provincia finita de sentido entre otras, el mundo de la vida se presenta como la realidad preeminente a la que, en el curso del día, volvemos reiteradamente. Nos desenvolvemos en diferentes mundos como el mundo de los sueños, el mundo de la fantasía, el mundo de la experiencia religiosa, el mundo de la contemplación científica, el mundo de los juegos y el mundo del enfermo, $y$, dentro de ciertos límites, podemos aprehender estos ámbitos de sentido como modificaciones del mundo de la vida cotidiano. Que cada uno de estos mundos sea una provincia finita de sentido significa que todas las experiencias que engloba son consistentes en sí mismas y compatibles unas con otras, tienen su particular estilo vivencial o estilo de conocimiento, y enfatizan de determinada manera la realidad. Esas características varían según cada provincia de sentido,

\footnotetext{
7 Alfred Schutz, Collected Papers. I. The Problem of Social Reality, The Hague: Martinus Nijhoff, $1967^{2}$, p. 58.

${ }^{\prime}$ Ibid., p. 10; cf. L. Embree, The Schutzian Theory, pp. 95s, 131.
} 
y, por tanto, el estilo de conocimiento en el mundo de la contemplación científica será distinto del estilo del mundo de la vida9.

El mundo de la vida nos es dado sobre la base del acervo de saber que resulta de una sedimentación de experiencias y es en su mayor parte socialmente derivado. Consiste en un conjunto de tipificaciones y apresentaciones, instrumentos, instituciones, costumbres, y sistemas simbólicos ${ }^{10}$. Así, el ser humano se encuentra en una situación histórica que resulta de todas las experiencias previas organizadas en las posesiones habituales de este acervo: "Como Husserl, a cuyas investigaciones básicas la presente visión debe tanto, ha mostrado, todas las formas de reconocimiento e identificación [...] están basadas en un conocimiento generalizado del tipo de estos objetos o del estilo típico en que se manifiestan"11. Schutz considera, siguiendo a Husserl, que la cultura presupone una constitución primaria y una constitución secundaria o pasiva, y Embree observa que "los objetos culturales son constituidos biográficamente en el hábito para los individuos e históricamente en tradiciones para grupos y el resto de lo que Schutz dice puede ser visto desde esta perspectiva"12. Puesto que es experienciado como tipificado, el mundo encierra horizontes abiertos de experiencias y acciones típicas similares. Los aspectos típicos son en principio repetibles y predecibles por medio de una idealización. Schutz observa que la idealización supone que lo que ha sido válido hasta ahora seguirá siendo válido, y su correlato subjetivo supone que lo que se ha podido hacer hasta ahora será posible en el futuro. Ambas idealizaciones suponen la constancia de la estructura del mundo y de su modo de alteración y correlativamente la constancia de mi capacidad de obrar en el mundo ${ }^{13}$.

En el mundo de la vida se distinguen el mundo circundante inmediato (Umwelt) de nuestros asociados cercanos o consocios, el mundo concomitante mediato (Mitwelt) de nuestros contemporáneos más distantes y conocidos de modo indefinido con su creciente anonimato, y el mundo previo (Vorwelt) de nuestros predecesores ${ }^{14}$. La delimitación de estas esferas ofrece un fundamento

${ }^{9}$ Cf. A. Schutz, Collected Papers I, pp. 207-259.

${ }_{10}$ Cf. Alfred Schutz, Collected Papers. III. Studies in Phenomenological Philosophy, The Hague: Martinus Nijhoff, 1970, p. 132.

${ }_{11}$ Alfred Schutz, Collected Papers. II. Studies in Social Theory, The Hague: Martinus Nijhoff, 1964, p. 284

12 Ibid., p. 199.

13 Cf. A. Schutz, Collected Papers III, p. 116.

${ }^{14}$ Cf. Alfred Schutz, Der sinnhafte Aufbau der sozialen Welt. Eine Einleitung in die verstehende Soziologie, Frankfurt am Main: Suhrkamp, 1981 (1a. ed.: Viena: Springer, 1932), pp. 227-302. 
para diferenciar en el ámbito de las ciencias de la cultura o ciencias sociales en sentido amplio dos especies de ciencias: las ciencias sociales en sentido restringido o estricto y las ciencias históricas. Las ciencias sociales en sentido restringido conciernen a los contemporáneos, es decir, a los otros que, aunque no comparten un mismo lugar, participan de un mismo tiempo. En su análisis, Embree incluye la economía, la jurisprudencia, la lingüística, la ciencia política, la psicología social y la sociología. La otra especie está compuesta por las ciencias históricas que se refieren a los predecesores, es decir, a los otros que no comparten nuestro tiempo. Comprende la biografía y la historia (historia de la economía, el arte, el derecho, la música, la filosofía y la política) ${ }^{15}$.

\section{DEFINICIONES DE DISCIPLINAS}

Consideramos en este apartado algunas de las definiciones que Embree ofrece sobre las ciencias culturales ya sea mediante la interpretación de Schutz o recurriendo a de desarrollos propios. Puesto que el tema no es tratado siempre en forma exhaustiva, sino que es indicado para una elaboración ulterior, nos limitamos a algunos ejemplos con la finalidad de ilustrar una de las tres cuestiones destacadas por Embree en la constitución de una ciencia de la cultura.

La sociología se ocupa de los contemporáneos. Puede ser considerada como psicología social cuando se practica un individualismo metodológico y se comienza con el individuo que se relaciona con otros en lugar de un colectivismo metodológico que comienza con el grupo social al que pertenecen los individuos. Sobre la base de un análisis de textos dispersos, Embree concluye que "para Schutz los grupos son concretos y los individuos considerados aparte de su calidad de miembros son abstracciones", pero añade que "el gran énfasis de Schutz, sin embargo, es sobre los individuos y esto se advierte tal vez del modo más claro en relación al modo en que los individuos comprenden y/o influyen en los asociados cercanos, los contemporáneos, los predecesores y los sucesores, $[\ldots]^{\prime 16}$. Puesto que elabora una "sociología comprensiva" (verstehende Soziologie) o "sociología interpretativa (interpretative sociology), que converge con lo

${ }^{15}$ Cf. L. Embree, The Schutzian Theory of Cultural Sciences, p. 17.

16 Ibid., p. 43. 
que en Estados Unidos se denomina psicología social, Schutz puede ser calificado, más que como un sociólogo, como un psicólogo social.

Sobre la base de afirmaciones dispersas, Embree intenta "construir lo que probablemente hubiera llegado a ser la teoría de Schutz sobre la ciencia política"17. Tiene en cuenta su familiaridad con las teorías políticas de Platón, Aristóteles, Agustín, Tomás de Aquino, Maquiavelo, Hobbes, Spinoza, Kant, Hegel, Marx y Nietzsche, y sostiene que la ciencia política es ante todo una ciencia social en sentido estrecho en que se investiga a contemporáneos, es decir, a seres humanos que necesariamente comparten un tiempo, pero no un lugar. Por eso se debe establecer lo que distingue positivamente a la ciencia política de otras ciencias sociales en esta significación estrecha. Hay "relaciones de poder político"18 en la estructura del mundo social. Nacemos dentro de esta organización política que tiene una jerarquía de gobernantes y gobernados. Schutz considera que las colectividades sociales con estas relaciones no están incluidas dentro de la provincia de sentido finita de la vida cotidiana sino que son constructos del sentido común que la trascienden y que solo se aprehenden a través de símbolos que pertenecen a esa realidad cotidiana. Por ejemplo, los imperios tempranos se comprendieron como representantes del orden cósmico, de modo que la jerarquía de gobernantes y gobernados se correlaciona con la jerarquía de los cuerpos celestes. La conclusión del análisis de Embree es que la ciencia política "se ocupa de colectividades compuesta de tipos ideales especialmente de funcionarios y de sus relaciones de poder, y que estos tipos forman una provincia finita de sentido trascendente a la que se refieren símbolos y el mito en la vida cotidiana y que al menos a veces se toman como representantes de un orden cósmico"19.

Schutz afirma que "una antropología filosófica, en el sentido continental europeo, es la ciencia del hombre, y no una ciencia de la cultura primitiva, que, en contraste con la antropología, se llama etnología en Europa"20. Ateniéndose a esta caracterización, Embree procura mostrar cómo se podría desplegar una teoría schutziana de la antropología cultural ajustada a la indicación de que no es una antropología filosófica, tiene la denominación alternativa de etnología, y

17 Ibid., p. 33.

18 A. Schutz, Collected Papers II, p. 249.

19 L. Embree, The Schutzian Theory of Cultural Sciences, p. 36. Los constructos y tipos ideales se tratan en el apartado siguiente.

${ }^{20}$ A. Schutz, Collected Papers II, p. 205. 
encuentra su tema en la cultura primitiva. Schutz no presenta una teoría explícita de la antropología cultural, pero ofrece los inicios de una ciencia de la cultura que puede articularse según la trilogía de la definición, los conceptos básicos y los métodos distintivos. Esta disciplina se ocupa del "patrón cultural de una vida grupal", esto es, de una noción que, según Schutz, tiene que ver con "todas las peculiares variaciones, instituciones y sistemas de orientación y conducción (tales como maneras populares (folkways), usos, leyes, hábitos, costumbres, etiqueta, modas) que, en la opinión común de los sociólogos de nuestro tiempo, caracterizan - si no constituyen- a cualquier grupo social en un momento de su historia"21. Embree continúa la definición por medio de eliminaciones. Puesto que esta es colectiva, no se trata de una ciencia psicológica que se ocupa de vidas individuales. Tampoco es una ciencia histórica porque las sociedades primitivas, si bien han tenido un pasado, no tienen de él un registro escrito y solo son accesibles por medio de la arqueología. Se trata de una ciencia social en la significación estrecha del término.

Aunque no sea clasificada por Schutz de esta manera, Embree considera que, puesto que no es una ciencia formal ni una ciencia natural al modo del conductismo, la psicología fenomenológica no puede ser otra cosa que una ciencia cultural. Schutz admite un enfoque que abstrae al individuo de la sociedad. Aunque afirma que un curso de conciencia considerado aisladamente es una "abstracción ficticia [...] efectuada meramente en aras de una presentación más clara de los problemas involucrados"22, reconoce la legitimidad de proceder mediante un solipsismo metodológico "como si un individuo aislado tuviera una experiencia del mundo de la naturaleza desconectado de sus congéneres"23.

\section{CONCEPTOS BÁSICOS}

Al igual que en el caso de las definiciones disciplinares, nos limitamos a algunos ejemplos. Pero antes es necesario avanzar en un terreno que se cruza con el de la metodología y esclarecer el modo en que se llega a los conceptos.

${ }^{21}$ Ibid., p. 92.

22 A. Schutz, Collected Papers I, p. 218. p. 135.

${ }^{23}$ A. Schutz, Collected Papers V. Phenomenology and the Social Sciences, Dordrecht: Springer, 2011, 
Esto significa, en primer lugar, distinguir las construcciones del sentido común y las construcciones de un análisis científico de la cultura.

Las construcciones del sentido común, que caracterizan al ser humano en la vida cotidiana, resultan del hecho de que este mundo de la vida es experienciado por el pensamiento precientífico en el modo de la tipicidad, y de que las acciones que se realizan en él tienen los sentidos subjetivos que les confieren los actores. Estos sentidos se captan mediante la elaboración de patrones típicos de motivos, fines, actitudes y personalidades. Las construcciones se refieren a elementos subjetivos, esto es, a la comprensión que los actores sociales tienen de lo que hacen. Schutz recuerda que Max Weber destaca como tema central la comprensión del sentido que el actor confiere a su acción, y observa que todo intento de determinar lo que la acción y el sentido son, y el modo en que es posible la comprensión de ese sentido, conduce inmediatamente a problemas que preocupaban a Husserl.

El sentido común organiza sus construcciones en torno del "aquí" de los seres humanos, y, por tanto, tiene como punto de partida lo que Schutz denomina "reciprocidad de perspectivas". La actitud natural en el mundo de la vida cotidiana se caracteriza por dos axiomas fundamentales: (i) la existencia incuestionable de congéneres dotados de conciencia; y (ii) la posibilidad por principio para ellos de experienciar los objetos del mundo de la vida de una manera similar a la mía. El segundo axioma admite diferencias en virtud de la variada articulación espacial, biográfica o sociocultural del mundo, pero, para fines prácticos, estas diferencias son superadas por dos construcciones. Una es la idealización de la intercambiabilidad de los puntos de vista según la cual ocupar el lugar del otro permite tener una experiencia del mundo sustancialmente similar a la suya. La otra construcción es la idealización de la congruencia de los sistemas de relevancia según la cual la diferencia entre estos sistemas carece de significación tanto para mí como para los otros en nuestros propósitos prácticos ${ }^{24}$. Ambas idealizaciones componen la tesis general de la reciprocidad de perspectivas $^{25}$.

${ }^{24}$ Schutz destaca tres tipos de relevancia: i) la relevancia temática de los objetos que atrae nuestra atención; ii) la relevancia interpretativa de los elementos del acervo de conocimiento que permiten hacer frente a los objetos; y iii) la relevancia motivacional que explica la acción a través de sus metas (motivospara) y las fuerzas impulsoras (motivos-porque).

25 Cf. A. Schutz, Collected Papers I, pp. $11 \mathrm{~s}$. 
Las construcciones que el científico social elabora sobre los patrones de la interacción humana se diferencian de las construcciones del sentido común desarrolladas en la vida cotidiana. Aquí se plantea el problema de la formación de conceptos objetivos verificables sobre la triple base proporcionada por (i) la observación de estructuras de sentido subjetivas, (ii) los constructos ya establecidos por los actores en la escena social y (iii) los procedimientos de la respectiva ciencia cultural. Estos conceptos objetivos no se organizan en torno del "aquí" del científico y no tienen su punto de partida en la reciprocidad de perspectivas. No obstante, tienen que estar fundadas en los constructos del sentido común que han sido previamente formados en la vida cotidiana. A diferencia de las ciencias de la naturaleza que no se atienen a una preselección, las ciencias sociales estudian hechos y datos que son preinterpretados y que tienen estructuras de relevancia intrínseca: "Las construcciones científicas formadas en el segundo nivel [...] son construcciones típicas objetivas e ideales, $y$, en cuanto tales, de un tipo diferente de aquellas desarrolladas en el primer nivel del pensamiento de sentido común al que substituye"26.

Embree considera que un problema emerge en la visión de Schutz porque "no está claro lo que son y a qué se refieren los constructos de la vida cotidiana expresados en el idioma vernáculo"27. Observa que es necesario tratar el tema de los constructos que no son específicos o generales, es decir, el caso de una realidad individual y única que implica una tipificación singular. Además del constructo cultural-científico, que es un objeto ideal, y el constructo del sentido común, que es también una idealidad - aunque vaga al modo del tipo empírico husserliano-, se encuentra la realidad del caso individual concreto que exhibe también su peculiar tipicidad asociada con una manera habitual de comportarse. En una corrección significativa, Embree señala que "[p]robablemente porque está interesado sobre todo en aquello a lo que se refieren los constructos culturalcientíficos, esto es, los constructos del sentido común, Schutz desafortunadamente no solo presta escasa atención a lo que he llamado objetos culturales básicos, sino que también enfatiza el modo en que los constructos del sentido común son generales y específicos"28.

\footnotetext{
${ }^{26}$ A. Schutz, Collected Papers I, p. 63; cf. p. 6.

27 L. Embree, The Schutzian Theory of Cultural Sciences, p. 201.

28 Ibid., p. 204. Embree menciona algunas indicaciones de Schutz sobre la tipicidad de los individuos. Cf. A. Schutz, Collected Papers II, p. 233; y Collected Papers III, p. 100.
} 
Schutz señala que son los conceptos básicos se relacionan con "la comprensión de sí y la comprensión de lo extraño, la posición de sentido y la interpretación de sentido, el símbolo y el síntoma, el motivo y el proyecto, la adecuación de sentido y la adecuación causal, y, sobre todo, la esencia de la formación de conceptos ideal-típica y con ella de la particular actitud de las ciencias sociales hacia su objeto"29. Los conceptos que se acaban de mencionar son válidos para todas las ciencias de la cultura. Junto a ellos se encuentran los conceptos básicos que son específicos de cada ciencia. Veamos algunos ejemplos.

Embree señala que, en relación con la jurisprudencia, Schutz menciona en sus escritos los conceptos de legislador, juez, abogado, veredicto, etc. Es fundamental el concepto de "norma básica" expuesta por Hans Kelsen como aquella regla general que determina el modo de establecer o anular otras normas, por ejemplo, mediante una decisión del parlamento, una creación de la costumbre o una decisión de los tribunales.

En lo que concierne a la antropología cultural, se trata para Embree de conceptos vinculados a los sistemas económicos como horticultura, pastoralismo, agricultura y modos de intercambio, al desarrollo humano como infancia, niñez, adolescencia y adultez, a la enfermedad y la curación, a los sistemas de parentesco y a los grupos sociales y su estratificación. Se añaden conceptos como autoridad, banda, clan, facción, feudos, control social, tribu, guerra, etc.

En el caso de la psicoterapia es necesaria una clara comprensión de conceptos básicos como terapista, cliente, práctica de curación, psiconeurótico, neurótico severo, estilos de prevención, terapia cognitiva, terapia de autocontrol, terapia interpersonal, terapia de conducta y terapia para la solución de problemas sociales.

\section{Metodología}

En la consideración de los métodos es necesario comenzar también, como en el caso de los conceptos básicos, con consideraciones generales sobre todas las ciencias de la cultura. Schutz señala que las ciencias culturales, al igual que las ciencias naturales, se desenvuelven con modelos. El científico cultural observa patrones típicos de acción y sobre esa base construye modelos en términos de

${ }^{29}$ A. Schutz, Der sinnhafte Aufbau, p. 9. 
un sistema de tipos ideales mutuamente coordinados referidos a la acción, los roles, las relaciones, las situaciones y los productos. La idealización en las ciencias sociales tiene un papel análogo al que Husserl ha descrito en relación con las ciencias de la naturaleza. Sin embargo, en lugar de una matematización de las formas, Schutz se refiere a una "tipología de 'plenitudes'"30. Las idealizaciones se presentan como tipologías, y también surge el peligro de interpretarlas como el verdadero ser en lugar de ver ellas el resultado de un método. Dada la complejidad del estrato fundante, es fácil perder la referencia retrospectiva de los tipos a la actividad subjetiva de los individuos.

Schutz sostiene que "una teoría que tiende a explicar la realidad social tiene que desarrollar procedimientos particulares extraños a las ciencias naturales a fin de concordar con la experiencia del mundo social inherente al sentido común" 31 . Estos procedimientos delinean cursos de acción típicos que se basan en la observación de la acción humana e incluyen el homúnculo o títere, es decir, un actor imaginario. El títere está dotado de una conciencia ficticia con sus nociones, propósitos, metas y sistemas de relevancia que se suponen invariantes. Se asigna a esta conciencia ficticia una serie de motivos invariantes que se apoyan en la experiencia previa. El motivo-para es la meta que mueve la acción en función del interés por un estado de cosas que se ha de efectuar, y que se representa como proyecto por medio de la fantasía. Hay también motivos-porque referidos a experiencias pasadas que llevan a actuar de cierta manera. La manera de alcanzar la meta $-\mathrm{y}$ hay diferentes maneras de llegar a ella-, es determinada por un motivo-porque que depende de la historia vital sedimentada. Así, las motivaciones - para se fundan en un complejo de motivos- porque que configuran la situación biográfica de quien actúa, esto es, su actitud. Además, el títere se relaciona con otros títeres construidos de la misma manera. Las acciones y reacciones son ficticias porque no se originan en una conciencia viviente como manifestaciones de su espontaneidad.

Por tanto, mediante procedimientos especiales, se extrae del mundo de la vida intersubjetivo un modelo de este ámbito de acción social: "[...] el científico reemplaza los seres humanos o actores en el escenario social por marionetas creadas por él mismo. Lo que llamo 'marionetas' corresponde al término técnico

\footnotetext{
${ }^{30}$ A. Schutz, Collected Papers I, p. 138.

${ }^{31}$ Ibid., p. 58.
} 
'tipos ideales' que Weber ha introducido en las ciencias sociales"32. En relación con la marioneta o actor-modelo se imaginan patrones típicos para sus cursos de acción y "una conciencia restringida a no contener nada más que los elementos relevantes para la ejecución del los patrones del curso de acción que se encuentra bajo observación, y por tanto, relevantes para el problema que se encuentra bajo inspección para el científico"33.

Los constructos típico-ideales difieren de los constructos de primer orden por su carácter ideal y porque implican hipótesis fundamentales que pueden ser puestas a prueba. Un ejemplo es el principio de utilidad en la economía clásica según el cual los tipos ideales deben ser construidos como si los actores orientaran toda su actividad a alcanzar la máxima utilidad con el mínimo costo. Al respecto, Schutz escribe: "Cada rama de las ciencias sociales que haya alcanzado el estadio teórico contiene una hipótesis fundamental que a la vez define el campo de investigación y ofrece los principios regulativos para la construcción de un sistema de tipos ideales" ${ }^{34}$. Esto puede ejemplificarse d la siguiente manera.

La jurisprudencia se caracteriza metodológicamente por la formulación de leyes que emanan de un legislador y a las que quedan sujetas las personas que las cumplen o las transgreden. Esto requiere tribunales que las interpretan y agentes que se esfuerzan por implementarlas ${ }^{35}$. Asimismo, es importante el uso de tipos ideales. Embree interpreta que las leyes se refieren a acciones que pueden ser clasificadas según tipos ideales de cursos de acción.

En la antropología cultural, la observación participativa ocupa un lugar central en virtud del trabajo de campo por el cual el investigador vive durante un tiempo considerable entre sus informantes en la sociedad primitiva. Se requieren también determinados equipamientos y vestimentas, el aprendizaje del dialecto local o la ayuda de un intérprete local y un esfuerzo para entablar una relación con los informantes. Asimismo, es necesaria la guía de una teoría en el sentido de ideas generales que puedan aplicarse a los casos particulares. Embree observa que "una teoría schutziana de la antropología cultural tendrá que reconocer el

\footnotetext{
32 A. Schutz, Collected Papers II, p. 81.

33 A. Schutz, Collected Papers I, p. 40.

${ }^{34}$ Alfred Schutz, Collected Papers. IV, Dordrecht/Boston/London: Kluwer Academic Publishers, 1996, p. 23.

${ }^{35}$ Cf. A. Schutz, Collected Papers II, p. 276.
} 
nivel de la teorización"36 y destaca la importancia que al respecto tienen los "motivos-para" descritos por Schutz.

El método de la psicoterapia se relaciona con la necesidad de lograr una efectiva interacción entre el terapista y el cliente. Embree destaca la necesidad de que ambos participantes compartan la creencia en una teoría explicativa adecuada. Esta creencia es independiente de la verdad que pueda tener o no la teoría en cuestión.

En suma: más allá de lo elaborado por Schutz, Embree sugiere la necesidad de reconocer constructos de tercer nivel, esto es, constructos científico-teóricos que se apoyan en los constructos científico-culturales, a su vez sustentados en los constructos del sentido común. El tercer nivel configura una teoría de la ciencia limitada a una ciencia particular que se ocupa de la definición de la disciplina, sus conceptos básicos y procedimientos metodológicos.

\section{TEORÍA FILOSÓFICA DE LA CIENCIA}

Embree expresa la conveniencia de reconocer, en un estrato superior, constructos de cuarto orden en el ámbito de una teoría de la ciencia de carácter filosófico que no se limita a una de las disciplinas como sucede en el tercer nivel ${ }^{37}$. En este estadio superior se efectúa, pues, un examen de los postulados válidos para todas las ciencias culturales.

En todas las ciencias rige el postulado de racionalidad o consistencia lógica que implica el uso de la lógica formal y de esquemas interpretativos junto con la exigencia de un máximo de claridad y de distinción para todas sus proposiciones. Según Schutz, este postulado garantiza la validez objetiva de los constructos elaborados en las ciencias de la cultura. En contraste con el pensamiento ideológico y su reflejo en la ciencia, Schutz adhiere a la neutralidad axiológica propuesta por Max Weber, es decir, a la abstracción de juicios de valor: "La teorización científica [...] no sirve a ningún propósito práctico. Su meta no es dominar el mundo sino observarlo y posiblemente entenderlo"38. Por otro lado, Schutz distingue entre teoría pura y teoría aplicada: "La tarea de las ciencias sociales es estudiar la estructura más bien complicada de la realidad social [...].

\footnotetext{
${ }^{36}$ L. Embree, The Schutzian Theory of Cultural Sciences, p. 75.

${ }^{37}$ Cf. ibid, p. 18.

38 A. Schutz, Collected Papers I, p. 245.
} 
La tarea de la investigación empírica es aplicar los hallazgos teóricos en el estudio de grupos sociales y relaciones sociales concretas en un medio dado en un momento histórico dado"39. Embree pone como ejemplo el enfoque que tuvo Schutz sobre el tratamiento de los veteranos que regresaban de la Segunda Guerra Mundial ${ }^{40}$.

Otro postulado es el principio de relevancia. Schutz afirma: "El concepto de relevancia es el concepto central de la sociología y de las ciencias culturales"41. La teoría es aquella provincia finita de sentido que corresponde a la actitud del observador desinteresado. En ella, el estilo cognoscitivo que caracteriza a toda provincia finita de sentido adquiere un carácter particular. Consiste en el abandono del sistema de relevancias que prevalece en el mundo de la vida práctico, es decir, en la puesta entre paréntesis de la subjetividad corporal, del sistema de orientación en torno del cuerpo propio y del sistema de relevancias pragmáticas inherente al mundo de la vida. Las relevancias difieren para el actor en la escena social y para el científico: lo que es relevante para uno puede ser irrelevante para el otro. Se instaura un nuevo sistema de relevancias que depende de la definición de los elementos del mundo que son efectiva y potencialmente importantes para el problema en consideración. El problema surge del estado actual de la ciencia y se trata según la metodología de esa ciencia. De este modo emergen también los constructos de segundo orden.

Además, en todas las ciencias de la cultura tienen validez los postulados de adecuación y de interpretación subjetiva. Se trata de postulados que sirven para anclar los constructos de segundo orden de las ciencias de la cultura en los constructos de primer orden por medio de los cuales los actores comprenden su mundo de la vida. Aseguran el contacto con la realidad evitando que las construcciones sean arbitrarias. Sobre el postulado de adecuación, Schutz señala que "cada término de un modelo científico de acción humana debe ser construido de tal modo que un acto humano efectuado dentro del mundo de la vida de un actor individual de la manera indicada por el constructo típico sea comprensible

${ }^{39}$ A. Schutz, Collected Papers IV, p. 150.

${ }^{40}$ Cf. L. Embree, The Schutzian Theory of Cultural Sciences, p. 16; y A. Schutz, Collected Papers II, pp. $116 \mathrm{ss}$.

${ }^{41}$ A. Schutz, Collected Papers IV, p. 3. 
por el actor y sus compañeros en términos del pensamiento de sentido común inherente a la vida cotidiana"42.

El postulado de interpretación subjetiva establece que toda situación o acción tiene un sentido subjetivo para la persona involucrada y un sentido objetivo en la interpretación de otra persona. Significa que "todas las explicaciones científicas del mundo social pueden, y para ciertos propósitos deben, referirse al sentido subjetivo de las acciones de los seres humanos a partir de los cuales se origina la realidad social"43. Esto remite a lo que Max Weber denominó postulado del sentido subjetivo, pero Schutz estima que esta terminología es desafortunada porque los así llamados sentidos objetivos son también relativos a algún tipo de sujeto ${ }^{44}$. Por su parte, Embree observa críticamente respecto de Schutz que, en lugar de "subjetivo" y "objetivo" -términos que se asocian cognoscitivamente con la idea de confiabilidad-, es mejor hablar de "interno a un grupo" (insider) y de "externo a un grupo" (outsider). El sentido se asocia con la interpretación, y, por tanto, se da (i) la interpretación interna de sentido común relativa al actor, (ii) la interpretación externa de sentido común relativa al socio interactuante, (iii) la interpretación externa del observador en el plano del sentido común, (iv) la interpretación externa del científico social y (v) la interpretación externa del filósofo. Por tanto, una ciencia cultural y la filosofía procuran una interpretación externa de las interpretaciones internas del sentido común ${ }^{45}$.

Embree escribe: "Mi tesis es que la metodología o teoría de la ciencia es el lugar en que quienes se dedican a las ciencias humanas y los filósofos pueden encontrarse" 46 . Por un lado, quienes se ocupan de las ciencias de la cultura se dedican a la metodología con el objeto de desarrollar sus categorías y técnicas de investigación. Por eso Schutz sostiene que sus representantes "trataron de superar las dificultades con las que se encontraban en el tratamiento de los problemas concretos de las ciencias sociales de los que se ocupaban forjando sus propios instrumentos metodológicos sin un intento de esclarecer la posición filosófica subyacente ${ }^{\prime 47}$. Por otro lado, teniendo en cuenta a Schutz como ejemplo de un filósofo que se ocupa de la metodología, Embree escribe: "Filósofos y

\footnotetext{
42 Alfred Schutz, "Positivist Philosophy and the Actual Approach of Interpretative Social Science. An Ineditum from 1953", Husserl Studies, vol. 14 (1998), p. 148.

${ }^{43}$ A. Schutz, Collected Papers I, p. 62.

${ }^{44}$ Cf. A. Schutz, Collected Papers II, pp. 227, 275.

45 Cf. L. Embree, The Schutzian Theory of Cultural Sciences, pp. $173 \mathrm{~s}$.

${ }^{46}$ Ibid., p. 105.

47 A. Schutz, "Positivist Philosophy", p. 125.
} 
científicos de la cultura pueden encontrarse en la metodología, pero no tienen que hacerlo. [...] El mejor acuerdo parece ser para ellos que cada uno persiga sus metas por separado y que se encuentren solo en la metodología cuando hay necesidad de ella"48. Este encuentro se hace necesario en virtud de las diversas perspectivas posibles sobre la acción de un actor, esto es, las que corresponden al actor mismo, al socio interactuante, al observador en la vida cotidiana, al científico social y al filósofo. Respecto de este escalonamiento, Embree afirma: "Cada perspectiva superior incluye en su alcance a las que se encuentran por debajo de ella"49. Por eso observa también que "[I]a teoría de la ciencia filosófica es la más amplia en alcance, pero la más alejada de los fenómenos concretos que son bases para toda la estructura"50.

La teoría de la ciencia filosófica no solo se ocupa de múltiples disciplinas culturales en un tratamiento global de sus metodologías, sino que considera las investigaciones del científico en las ciencias de la cultura $y$, a través de estas investigaciones, tiene que contemplar el sentido subjetivo de acciones y objetos culturales. El filósofo debe describir y analizar fenómenos de sentido que simplemente transcurren para la persona ingenua, es decir, "reactivar el proceso que ha construido los sedimentos de sentido" y "explicar las intencionalidades de las perspectivas de relevancia y de los horizontes de interés"51.

Embree señala que un análisis reflexivo del objeto como correlato de la acción subjetiva descubre tres clases de características posicionales noemáticas que corresponden a la creencia (existencia, inexistencia, existencia neutral), la valoración (bondad, maldad, neutralidad valorativa) y la volición (utilidad positiva, negativa o neutra). Aquellas características posicionales que se constituyen de una manera típica porque han sido constituidas por el hábito o la tradición son "características culturales". Se las excluye cuando el objeto es puesto como un mero objeto de la naturaleza. De este modo las ciencias naturales dejan a un lado el valor y el uso. O sea: todos los objetos tienen originariamente características culturales, y pueden ser especificados en términos de caracteres de creencia, valores y usos. Así, las ciencias de la cultura implican un género con tres subespecies: una ciencia cognoscitiva cuando se

\footnotetext{
${ }^{48}$ L. Embree, The Schutzian Theory of Cultural Sciences, p. 106.

${ }^{49}$ Ibid., p. 5.

50 Ibid., p. 81.

${ }^{51}$ A. Schutz, Collected Papers I, p. 136.
} 
enfatiza el estrato dóxico como en el caso de la historiografía, una ciencia vinculada al valor cuando se fomenta el estrato axiológico como en el caso del arte o la arquitectura, y una ciencia práctica cuando predomina el estrato valorativo como en la psiquiatría o la enfermería ${ }^{52}$.

El artículo mencionado al comienzo de este trabajo termina con la formulación de una aspiración a cuyo cumplimiento Embree mismo ha contribuido de manera decisiva mediante este análisis y ampliación de la teoría schutziana de la cultura: "Para los fenomenólogos filosóficos, los beneficios de comunicarse con los científicos culturales puede ser grande, especialmente si el quinto período está orientado hacia la cultura básica. No todos los problemas filosóficos han sido formulados y hay mucho que aprender. Espero vivir lo suficiente para ver si se confirman mis predicciones relativas a un quinto período de la fenomenología"53.

\section{REFERENCIAS BIBLIOGRÁFICAS}

EmbreE, Lester, "Cultural Disciplines", en Encyclopedia of Phenomenology, Dordrecht/Boston/London: Kluwer Academic Publishers, 1997, pp. 121123.

—, "La continuidad de la fenomenología: ¿un quinto período?", Escritos de Fenomenología 37-38 (2000) 17-29.

- Análisis reflexivo. Una primera introducción a la investigación fenomenológica, Morelia: Jitanjáfora, 2003.

-, The Schutzian Theory of Cultural Sciences, Cham/Heidelberg/New York/Dordrecht/London: Springer, 2017.

EmbreE, Lester / J. N. MohanTY, "Preface", en Encyclopedia of Phenomenology, Dordrecht/Boston/London: Kluwer Academic Publishers, 1997, pp. 1-10.

ScHuTZ, Alfred, Collected Papers. I. The Problem of Social Reality, The Hague: Martinus Nijhoff, $1967^{2}$.

-, Collected Papers. II. Studies in Social Theory, The Hague: Martinus Nijhoff, 1964.

52 Cf. Lester Embree, "Cultural Disciplines", Encyclopedia of Phenomenology, pp. 121-123, y Análisis reflexivo. Una primera introducción a la investigación fenomenológica, Morelia: Jitanjáfora, 2003, pp. 316335.

\footnotetext{
${ }^{53}$ L. Embree, "La continuación de la fenomenología", p. 29.
} 
-, Collected Papers. III. Studies in Phenomenological Philosophy, The Hague: Martinus Nijhoff, 1970.

-, Collected Papers. IV. Dordrecht/Boston/London: Kluwer Academic Publishers, 1996.

-, Collected Papers V. Phenomenology and the Social Sciences, Dordrecht: Springer, 2011.

-, Der sinnhafte Aufbau der sozialen Welt. Eine Einleitung in die verstehende Soziologie, Frankfurt am Main: Suhrkamp, 1981 [1a. ed.: Viena: Springer, 1932].

-, "Positivist Philosophy and the Actual Approach of Interpretative Social Science. An Ineditum of Alfred Schutz from 1953", Husserl Studies, vol. 14 (1998) 123-149. 